\title{
Neutrino-antineutrino pair production by a photon in a dense matter
}

\author{
A. E. Lobanov* \\ Moscow State University, Department of Theoretical physics, 119992 Moscow, Russia
}

\begin{abstract}
The possibility of radiative effects that are due to interaction of fermions with a dense matter is investigated. Neutrino-antineutrino photo-production is studied. The rate of this process is calculated in the Furry picture. It is demonstrated that this effect does not disappear even if the medium refractive index is assumed to be equal to unity. The rate obtained strongly depends on the polarization states of the particles involved. This leads to evident spatial asymmetries, which may have certain consequences observable in astrophysical and cosmological studies.
\end{abstract}

A Dirac massive neutrino has non-trivial electromagnetic properties. In particular, it possesses a non-zero magnetic moment [1]. Therefore a Dirac massive neutrino propagating in a dense matter can emit electromagnetic radiation due to the weak interaction with background fermions [2, 3]. The phenomenon was called the neutrino spin light in analogy with the effect, related with the synchrotron radiation power depending on the electron spin orientation (see [4]). The properties of spin light were investigated in the quasi-classical approach [2, 3, 5], and basing upon the consistent quantum theory [6, 7, 8].

However this is not the only effect caused by the non-trivial electromagnetic properties of neutrino. In the present work we investigate the possibility of $v \bar{v}$ pair production by a photon. As is evident from the following account, such process in a dense matter is available even if the refractive index of the medium is assumed to be equal to unity, i.e., the photon dispersion law is the vacuum one.

It is well known that the process $\gamma \rightarrow v+\bar{v}$ is kinematically possible whenever the photon four momentum $k^{\mu}=\left\{k^{0}, \mathbf{k}\right\}$ is time-like even if neutrinos are assumed to be massless. This process,

\footnotetext{
*E-mail: lobanov@phys.msu.ru
} 
called "plasmon decay", can really take place in stars (see, e.g., [9] and references therein). Unlike the "plasmon decay", the reaction under investigation is due to the modification of the neutrino dispersion law caused by the coherent interaction of neutrino with a dense matter.

When the interaction of neutrino with the background fermions is considered to be coherent, the propagation of neutrino in matter is described by the Dirac equation with the effective potential [10, 11]. In what follows, we restrict our consideration to the case of a homogeneous and isotropic medium. Then in the framework of the minimally extended standard model, the form of this equation is uniquely determined by the assumptions similar to those adopted in [12]:

$$
\left(i \hat{\partial}-\frac{1}{2} \hat{f}\left(1+\gamma^{5}\right)-m_{v}\right) \Psi_{v}=0 .
$$

The function $f^{\mu}=$ const is a linear combination of fermion currents and polarizations. The quantities with hats denote scalar products of the Dirac matrices with 4-vectors, i.e., $\hat{a} \equiv \gamma^{\mu} a_{\mu}$.

If the medium is at rest and unpolarized then $\mathbf{f}=0$. The component $f^{0}$ calculated in the first order of the perturbation theory is as follows [13, 14, 15]:

$$
f^{0}=\sqrt{2} G_{\mathrm{F}}\left\{\sum_{f}\left(I_{e v}+T_{3}^{(f)}-2 Q^{(f)} \sin ^{2} \theta_{\mathrm{W}}\right)\left(n_{f}-n_{\bar{f}}\right)\right\} .
$$

Here, $n_{f}, n_{\bar{f}}$ are the number densities of background fermions and anti-fermions, $Q^{(f)}$ is the electric charge of the fermion, and $T_{3}^{(f)}$ is the third component of the weak isospin for the left-chiral projection of it. The parameter $I_{e v}$ is equal to unity for the interaction of electron neutrino with electrons. In other cases $I_{e v}=0$. Summation is performed over all fermions of the background.

In what follows we use solutions of equation (10) in which quantum numbers of the problem are the kinetic momentum components of neutrino, related to its group 4-velocity $u^{\mu}$ by the relations $q^{\mu}=m_{v} u^{\mu}, q^{2}=m_{v}^{2}$. This choice can be justified, since it is the particle kinetic momentum that can be really observed.

When the medium is at rest and unpolarized then the orthonormalized system of such solutions is [6, 7]:

$$
\Psi(x)=\frac{\left|\Delta_{q \zeta}\right|}{\sqrt{2 q^{0}}} e^{-i\left(q^{0}+f^{0} / 2\right) x^{0}} e^{i \mathbf{q} \mathbf{x} \Delta_{q \zeta}}\left(\hat{q}+m_{v}\right)\left(1-\zeta \gamma^{5} \hat{S}_{t p}\right) \psi^{0},
$$

where $\Delta_{q \zeta}=1+\zeta f^{0} / 2|\mathbf{q}|$, and

$$
S_{t p}^{\mu}=\frac{1}{m_{v}}\left\{|\mathbf{q}|, q^{0} \mathbf{q} /|\mathbf{q}|\right\},
$$

i. e., the eigenvalues $\zeta= \pm 1$ determine the helicity of the particle. Here $\psi^{0}$ is an arbitrary normalized constant bispinor. 
It is clear that the relation of the canonical momentum $\mathbf{P}$ to the kinetic momentum $\mathbf{q}$ is determined by the formula

$$
\mathbf{P}=\mathbf{q} \Delta_{q \zeta},
$$

and the particle energy is given by the relation

$$
\varepsilon=q^{0}+f^{0} / 2=\sqrt{\mathbf{q}^{2}+m_{v}^{2}}+f^{0} / 2
$$

It is seen that if the energy is expressed in terms of the kinetic momentum, then it does not depend on the particle helicity, while the particle canonical momentum is a function of the helicity.

Consider the neutrino-antineutrino pair photo-production process. The formula for the neutrino pair creation probability by a polarized photon with the vacuum dispersion law is

$$
\begin{gathered}
P=-\frac{1}{2 k^{0}} \int d^{4} x d^{4} y \int \frac{d^{4} p d^{4} q}{(2 \pi)^{6}} \delta\left(p^{2}-m_{v}^{2}\right) \delta\left(q^{2}-m_{v}^{2}\right) \\
\times \operatorname{Sp}\left\{\Gamma_{\mu}(x) \varrho_{\bar{v}}\left(x, y ; p, \zeta_{\bar{v}}\right) \Gamma_{v}(y) \varrho_{v}\left(y, x ; q, \zeta_{v}\right)\right\} \varrho_{p h}^{\mu v}(y, x ; k) .
\end{gathered}
$$

Here, $\varrho_{v}\left(y, x ; q, \zeta_{v}\right), \varrho_{\bar{v}}\left(x, y ; p, \zeta_{\bar{v}}\right)$ are the neutrino and antineutrino density matrices respectively, $\varrho_{p h}^{\mu \nu}(y, x ; k)$ is the initial photon density matrix, $\Gamma^{\mu}=-\sqrt{4 \pi} \mu_{0} \sigma^{\mu v} k_{v}$ is the vertex function, where $\mu_{0}$ is the anomalous magnetic moment of the neutrino. The density matrices of longitudinally polarized antineutrino and neutrino in the unpolarized matter at rest constructed with the use of the solutions (3) of equation (1) have the form

$$
\begin{aligned}
& \varrho_{\bar{v}}\left(x, y ; p, \zeta_{\bar{v}}\right)=\frac{1}{2} \Delta_{p \zeta_{\bar{v}}}^{2}\left(\hat{p}-m_{v}\right)\left(1-\zeta_{\bar{v}} \gamma^{5} \hat{S}_{t p}(p)\right) e^{i\left(x^{0}-y^{0}\right)\left(p^{0}-f^{0} / 2\right)-i(\mathbf{x}-\mathbf{y}) \mathbf{p} \Delta_{p \zeta}}, \\
& \varrho_{v}\left(y, x ; q, \zeta_{v}\right)=\frac{1}{2} \Delta_{q \zeta_{\nu}}^{2}\left(\hat{q}+m_{v}\right)\left(1-\zeta_{v} \gamma^{5} \hat{S}_{t p}(q)\right) e^{i\left(x^{0}-y^{0}\right)\left(q^{0}+f^{0} / 2\right)-i(\mathbf{x}-\mathbf{y}) \mathbf{q} \Delta_{q \zeta_{\nu}}} .
\end{aligned}
$$

After integrating with respect to coordinates we obtain the expression for the transition rate under investigation:

$$
W=\frac{\mu_{0}^{2}}{k^{0}} \int \frac{d^{4} p d^{4} q}{(2 \pi)} \delta\left(p^{2}-m_{v}^{2}\right) \delta\left(q^{2}-m_{v}^{2}\right) \delta\left(k^{0}-p^{0}-q^{0}\right) \delta^{3}\left(\mathbf{k}-\mathbf{p} \Delta_{p \zeta_{\bar{v}}}-\mathbf{q} \Delta_{q \zeta_{\nu}}\right) T(p, q),
$$

where

$$
\begin{aligned}
T(p, q) & =2 \Delta_{p \zeta_{\bar{\nu}}}^{2} \Delta_{q \zeta_{\nu}}^{2}\left\{(p k)(q k)+\zeta_{\bar{\nu}} \zeta_{v}\left[k^{0}|\mathbf{p}|-p^{0}(\mathbf{p k}) /|\mathbf{p}|\right]\left[k^{0}|\mathbf{q}|-q^{0}(\mathbf{q} \mathbf{k}) /|\mathbf{q}|\right]\right. \\
& \left.-g \zeta_{\bar{\nu}}(q k)\left[k^{0}|\mathbf{p}|-p^{0}(\mathbf{p k}) /|\mathbf{p}|\right]-g \zeta_{v}(p k)\left[k^{0}|\mathbf{q}|-q^{0}(\mathbf{q} \mathbf{k}) /|\mathbf{q}|\right]\right\} .
\end{aligned}
$$

Here $g= \pm 1$ denotes the sign of the initial photon circular polarization. 
After integrating over angular variables of antineutrino and $|\mathbf{p}|,|\mathbf{q}|$, we obtain the spectralangular distribution of the final neutrino

$$
\begin{gathered}
W=\zeta_{\bar{\nu}} \zeta_{v} \frac{\mu_{0}^{2}}{4 \pi k^{0}} \int \frac{d p^{0} d q^{0}}{|\mathbf{p} \| \mathbf{q}|} \delta\left(k^{0}-p^{0}-q^{0}\right) \operatorname{sign}\left(\Delta_{p \zeta_{\bar{\nu}}} \Delta_{q \zeta_{\nu}}\right) \\
\times \int d O \delta\left(\sqrt{|\mathbf{p}|^{2} \Delta_{p \zeta_{\bar{\nu}}}^{2}-\left(k^{0}-|\mathbf{q}| \Delta_{q \zeta_{\nu}} \cos \vartheta_{v}\right)^{2}}-|\mathbf{q}|\left|\Delta_{q \zeta_{\nu}}\right| \sin \vartheta_{v}\right) \\
\times\left[\left(f^{0} / 2\right)\left(\zeta_{\bar{\nu}} q^{0}|\mathbf{p}|+\zeta_{\nu} p^{0}|\mathbf{q}|\right)-k^{0} m_{v}^{2}-g\left(f^{0} / 2\right)\left(\zeta_{\bar{\nu}} \zeta_{v}|\mathbf{p} \| \mathbf{q}|+m_{v}^{2}+p^{0} q^{0}\right)\right]^{2},
\end{gathered}
$$

where

$$
|\mathbf{p}|=\sqrt{\left(p^{0}\right)^{2}-m_{v}^{2}}, \quad|\mathbf{q}|=\sqrt{\left(q^{0}\right)^{2}-m_{v}^{2}}
$$

$\vartheta_{v}$ is the angle between the direction of the neutrino propagation and the photon wave vector, and $d O=\sin \vartheta_{v} d \vartheta_{v} d \varphi_{v}$ is the solid angle element.

Spectral-angular distribution of the final antineutrino can be found after substitution

$$
q^{0} \leftrightarrow p^{0}, \quad|\mathbf{q}| \leftrightarrow|\mathbf{p}|, \quad \zeta_{v} \leftrightarrow \zeta_{\bar{v}}, \quad \vartheta_{v} \rightarrow \vartheta_{\bar{v}},
$$

made in equation (11). Naturally, the kinetic momenta of neutrino $\mathbf{q}$ and antineutrino $\mathbf{p}$ are in a plane with the photon momentum $\mathbf{k}$. If $\operatorname{sign}\left(\Delta_{p \zeta_{\bar{\nu}}} \Delta_{q \zeta_{\nu}}\right)=1$, the kinetic momenta $\mathbf{p}, \mathbf{q}$ have opposite azimuthal angles. However if $\operatorname{sign}\left(\Delta_{p \zeta_{\bar{\nu}}} \Delta_{q \zeta_{v}}\right)=-1$, this vectors have the same azimuthal angles. It should be emphasized that this fact is rather unusual.

It is convenient to express the results of integrating over angular variables of neutrino in terms of dimensionless quantities. Introducing the notations

$$
\begin{array}{lll}
\eta=k^{0} / 2 m_{v}, & p^{0} / m_{v}=\eta+y, & q^{0} / m_{v}=\eta-y, \\
d=\left|f^{0}\right| / 2 m_{v}, & \bar{\zeta}_{\bar{v}, v}=\zeta_{\bar{v}, v} \operatorname{sign}\left(f^{0}\right), & \bar{g}=g \operatorname{sign}\left(f^{0}\right),
\end{array}
$$

we have

$$
\begin{gathered}
W_{\bar{g} \bar{\zeta}_{\bar{\nu}} \bar{\zeta}_{v}}=\frac{\mu_{0}^{2} m_{v}^{3}}{8 \eta^{2}} \int_{y_{-}}^{y_{+}} \frac{d y}{\sqrt{(\eta+y)^{2}-1} \sqrt{(\eta-y)^{2}-1}} \\
\times\left[d\left(\bar{\zeta}_{\bar{\nu}}(\eta-y) \sqrt{(\eta+y)^{2}-1}+\bar{\zeta}_{v}(\eta+y) \sqrt{(\eta-y)^{2}-1}\right)-2 \eta\right. \\
\left.-\bar{g} d\left(\bar{\zeta}_{\bar{\nu}} \bar{\zeta}_{v} \sqrt{(\eta+y)^{2}-1} \sqrt{(\eta-y)^{2}-1}+(\eta+y)(\eta-y)+1\right)\right]^{2} .
\end{gathered}
$$

For pair production the necessary condition on the photon energy is $\eta \geqslant 1$. In fact the threshold of the reaction can lie higher. So the integration limits $y_{ \pm}$in formula (14) are different for different values of parameter $d$.

When $d<\frac{1}{2}$ we have

$$
\begin{array}{ll}
y \in \varnothing & \eta \in\left[1, \eta_{1}\right), \\
y \in\left[-y_{0}, y_{0}\right] & \eta \in\left[\eta_{1}, \infty\right),
\end{array}
$$


if $\bar{\zeta}_{\bar{v}}=1, \bar{\zeta}_{v}=1$,

$$
y \in \varnothing \quad \eta \in[1, \infty),
$$

if $\bar{\zeta}_{\bar{v}}=-1, \bar{\zeta}_{v}=-1$

$$
y \in \varnothing \quad \eta \in[1, \infty),
$$

if $\bar{\zeta}_{\bar{v}}=-1, \bar{\zeta}_{v}=1$, and

$$
y \in \varnothing \quad \eta \in[1, \infty),
$$

if $\bar{\zeta}_{\bar{v}}=1, \bar{\zeta}_{v}=-1$.

When $1 / 2 \leqslant d \leqslant 1$ we have

$$
\begin{array}{ll}
y \in \varnothing & \eta \in\left[1, \eta_{1}\right), \\
y \in\left[-y_{0}, y_{0}\right] & \eta \in\left[\eta_{1}, \eta_{2}\right), \\
y \in[-(\eta-1), \eta-1] & \eta \in\left[\eta_{2}, \infty\right),
\end{array}
$$

if $\bar{\zeta}_{\bar{v}}=1, \bar{\zeta}_{v}=1$,

$$
y \in \varnothing \quad \eta \in[1, \infty),
$$

if $\bar{\zeta}_{\bar{v}}=-1, \bar{\zeta}_{v}=-1$,

$$
\begin{array}{ll}
y \in \varnothing & \eta \in\left[1, \eta_{2}\right), \\
y \in\left[-(\eta-1),-y_{0}\right] & \eta \in\left[\eta_{2}, \infty\right),
\end{array}
$$

if $\bar{\zeta}_{\bar{v}}=-1, \bar{\zeta}_{v}=1$, and

$$
\begin{array}{ll}
y \in \varnothing & \eta \in\left[1, \eta_{2}\right), \\
y \in\left[y_{0}, \eta-1\right] & \eta \in\left[\eta_{2}, \infty\right),
\end{array}
$$

if $\bar{\zeta}_{\bar{v}}=1, \bar{\zeta}_{v}=-1$.

In the case of high matter density $(d>1)$ we have

$$
y \in[-(\eta-1), \eta-1] \quad \eta \in[1, \infty),
$$

if $\bar{\zeta}_{\bar{v}}=1, \bar{\zeta}_{v}=1$,

$$
\begin{array}{ll}
y \in[-(\eta-1), \eta-1] & \eta \in\left[1, \eta_{1}\right), \\
y \in\left[-(\eta-1),-y_{0}\right] \cup\left[y_{0}, \eta-1\right] & \eta \in\left[\eta_{1}, \eta_{2}\right), \\
y \in \varnothing & \eta \in\left[\eta_{2}, \infty\right),
\end{array}
$$

if $\bar{\zeta}_{\bar{v}}=-1, \bar{\zeta}_{v}=-1$

$$
\begin{array}{ll}
y \in[-(\eta-1), \eta-1] & \eta \in\left[1, \eta_{2}\right), \\
y \in\left[-(\eta-1), y_{0}\right] & \eta \in\left[\eta_{2}, d\right), \\
y \in\left[-(\eta-1),-y_{0}\right] & \eta \in[d, \infty) .
\end{array}
$$


if $\bar{\zeta}_{\bar{v}}=-1, \bar{\zeta}_{v}=1$, and

$$
\begin{aligned}
& y \in[-(\eta-1), \eta-1] \quad \eta \in\left[1, \eta_{2}\right), \\
& y \in\left[-y_{0}, \eta-1\right] \quad \eta \in\left[\eta_{2}, d\right) \text {, } \\
& y \in\left[y_{0}, \eta-1\right] \quad \eta \in[d, \infty) \text {. }
\end{aligned}
$$

if $\bar{\zeta}_{\bar{v}}=1, \bar{\zeta}_{v}=-1$.

Here

$$
\eta_{1}=\frac{1+d^{2}}{2 d}, \quad \eta_{2}=\frac{d^{2}}{2 d-1}, \quad y_{0}=\frac{|\eta-d| \sqrt{\eta-\eta_{1}}}{\sqrt{\eta-d / 2}} .
$$

It is easy to verify that if parameter $\bar{\zeta}_{v}=-1$ for the generated neutrino, then $\Delta_{q \bar{\zeta}_{v}}<0$. In a similar way, if parameter $\bar{\zeta}_{\bar{v}}=-1$ for generated antineutrino, then $\Delta_{p \bar{\zeta}_{\bar{\nu}}}<0$. Therefore, such particles have $p^{0}<m_{v} \sqrt{1+d^{2}}$, or $q^{0}<m_{v} \sqrt{1+d^{2}}$, i.e., their energies lie lower than the spin light emission threshold [6, 7]. Since the condition $\bar{\zeta}=-1$ is necessary for photon emission by a neutrino in matter, the cascade process of the form

$$
\gamma \rightarrow v+\bar{v} \rightarrow v+\bar{v}+\gamma
$$

is impossible in our model.

The transition rate under investigation is defined as

$$
W_{\bar{g} \bar{\zeta}_{\bar{\zeta}} \bar{\zeta}_{v}}=\frac{\mu_{0}^{2} m_{v}^{3}}{12 \eta^{2}}\left(J\left(y_{+}\right)-J\left(y_{-}\right)\right) .
$$

Here

$$
\begin{gathered}
J(y)=d \eta\left(4 d \eta^{2}+d+6 \bar{g} \eta\right) E(\chi, s)-\eta\left(2 d^{2}-3\right) F(\chi, s) \\
+\frac{d}{2 \eta}\left(2 d\left(y^{2}-\eta^{2}\right)-3 d-6 \bar{g} \eta\right) \sin \chi \sqrt{\left((\eta-y)^{2}-1\right)\left((\eta+y)^{2}-1\right)} \\
+\bar{\zeta}_{\bar{\nu}} \bar{\zeta}_{\nu} d y\left(2 d\left(3 \eta^{2}-y^{2}\right)+3 d+6 \bar{g} \eta\right) \\
-2 \bar{\zeta}_{\nu} d\left(3 \eta-\bar{g} d\left(y^{2}-2 \eta^{2}-y \eta-1\right)\right) \sqrt{\left((\eta+y)^{2}-1\right)} \\
+2 \bar{\zeta}_{\bar{\nu}} d\left(3 \eta-\bar{g} d\left(y^{2}-2 \eta^{2}+y \eta-1\right)\right) \sqrt{\left((\eta-y)^{2}-1\right)},
\end{gathered}
$$

where $F(\chi, s), E(\chi, s)$ are elliptic integrals [16] of the arguments

$$
\chi=\arcsin \frac{2 y \eta}{y^{2}+\eta^{2}-1}, \quad s=\sqrt{1-\eta^{-2}} .
$$

If we consider the case with $d \ll 1$, the following approximate expression can be obtained

$$
\begin{gathered}
W_{\bar{g} \bar{\zeta}_{\bar{\nu}} \bar{\zeta}_{v}} \approx \frac{\mu_{0}^{2} m_{v}^{3}}{16 \eta}\left(1+\bar{\zeta}_{\bar{v}}\right)\left(1+\bar{\zeta}_{v}\right)\left\{\ln \frac{1+\sqrt{1-\eta_{1} / \eta}}{1-\sqrt{1-\eta_{1} / \eta}}(1+\bar{g})\right. \\
\left.+\left[\frac{4}{3}\left(\frac{\eta}{\eta_{1}}\right)^{2} \sqrt{\left(1-\eta_{1} / \eta\right)^{3}}-2 \sqrt{1-\eta_{1} / \eta}+\ln \frac{1+\sqrt{1-\eta_{1} / \eta}}{1-\sqrt{1-\eta_{1} / \eta}}\right](1-\bar{g})\right\} .
\end{gathered}
$$


Near the reaction threshold, defined by the relation $\eta=\eta_{1}$, the transition rate is described by the formula

$$
W_{\bar{g} \bar{\zeta}_{\bar{\nu}} \bar{\zeta}_{v}} \approx \frac{\mu_{0}^{2} m_{v}^{3}}{8 \eta}\left(1+\bar{\zeta}_{\bar{v}}\right)\left(1+\bar{\zeta}_{\nu}\right)\left[\sqrt{1-\eta_{1} / \eta}(1+\bar{g})+\sqrt{\left(1-\eta_{1} / \eta\right)^{3}}(1-\bar{g})\right],
$$

and for $d \eta \gg 1$, by the formula

$$
W_{\bar{g} \bar{\zeta}_{\bar{\zeta}} \bar{\zeta}_{v}} \approx \frac{\mu_{0}^{2} m_{v}^{3}}{3} d^{2} \eta\left(1+\bar{\zeta}_{\bar{v}}\right)\left(1+\bar{\zeta}_{v}\right)(1-\bar{g}) .
$$

When $d \gtrsim 1, \eta \gg d$ the transition rate is described by the formula (33) as well.

Pairs produced have zero angular orbital momentum if $\bar{g}=1$ ("allowed" transition), and non-zero angular orbital momentum if $\bar{g}=-1$ ("forbidden" transition). Evidently, only high energy photons with the helicity sign opposite to the sign of the effective potential $(\bar{g}=-1)$ can effectively produce neutrino and antineutrino. For high energy photons with $\bar{g}=1$ pair production is suppressed.

With the use of the effective potential calculated in the first order of the perturbation theory (2) the following conclusions can be made. Let us discuss, as an example, the neutron medium. The sign of the effective potential is negative in this case, so only right-handed polarized photons of high energy can effectively interact with such medium. In the ultra-relativistic limit, (here we use gaussian units), we have for the rate of pair production

$$
W_{\bar{g} \bar{\zeta}_{\bar{\zeta}} \bar{\zeta}_{v}} \approx \frac{\alpha \varepsilon_{\gamma}}{192 \hbar}\left(\frac{\mu_{0}}{\mu_{\mathrm{B}}}\right)^{2}\left(\frac{G_{\mathrm{F}} n_{n}}{m_{e} c^{2}}\right)^{2}\left(1-\bar{\zeta}_{i}\right)\left(1+\bar{\zeta}_{f}\right)(1-\bar{g}) .
$$

Here $\varepsilon_{v}$ is the neutrino energy, $\mu_{\mathrm{B}}=e \hbar / 2 m_{e} c$ is the Bohr magneton, $\alpha$ is the fine structure constant, $m_{e}$ is the electron mass, and $G_{\mathrm{F}}$ is the Fermi constant. This formula is valid for neutrinos of different flavors.

As a result of the reaction the left-handed polarized particles essentially arise: an active lefthanded polarized neutrino and a practically "sterile" left-handed polarized antineutrino. Electron neutrino can react with neutron medium as follows

$$
v_{e}+n \rightarrow p^{+}+e^{-}
$$

and $v_{\mu}, v_{\tau}$ cannot interact with the medium.

It is very interesting to consider the limit $m_{v} \rightarrow 0$ in eq. (14), when the neutrino anomalous magnetic moment $\mu_{0}$ is supposed to be constant. We have

$$
W_{\bar{g} \bar{\zeta}_{\bar{\nu}} \bar{\zeta}_{\nu}}=\frac{\mu_{0}^{2} k^{0}\left(f^{0}\right)^{2}}{24}\left[\left(1+\bar{\zeta}_{\bar{v}}\right)\left(1+\bar{\zeta}_{v}\right)(1-\bar{g})+\left(1-\bar{\zeta}_{\bar{v}}\right)\left(1-\bar{\zeta}_{v}\right)(1+\bar{g}) \Theta\left(\left|f^{0}\right|-2 k^{0}\right)\right] .
$$


It is evident that the right-hand side of eq. (35) vanishes if we use the standard two-component model of neutrino $\left(\zeta_{\bar{v}}=1, \zeta_{v}=-1\right)$. Hence the rate of the process is not equal to zero due to "sterile" states of neutrinos.

Recently [17] the possibility of electron-positron pair production by a photon was investigated in the framework of Standard Model Extension [18, 19, 20, 21] with axial-vector Lorentz breaking background. In this model the Dirac equation for an electron differ from (1) only by the term that can be gauge away. In the relativistic case, the formulas obtained in [17] for the rate of pair production coincide with those for "allowed" transition $(\bar{g}=1)$ in our model (see (31)). The transition rate decreases in the region of high energies as it must be in the renormalizable theory. It is significant that in our model based on the non-minimal interaction and, therefore, non-renormalizable, "forbidden" transitions play the main role at high energies.

In conclusion a few words about approximations used in our paper. It is possible to suppose that the interaction of neutrino with matter to be coherent if there are significant number of matter particles in the de Broglie cell of the neutrino. This condition leads to inequality

$$
\frac{n}{\gamma}\left(\frac{\hbar}{m_{v} c}\right)^{3} \gg 1 .
$$

If the matter number density is assumed to be $n \approx 10^{38} \mathrm{~cm}^{-3}$ which corresponds to the number density of a neutron star, we have

$$
\varepsilon_{v} \ll 10^{24}\left(\frac{m_{v}}{1 \mathrm{eV}}\right)^{-2} \mathrm{eV}
$$

Since the neutrino mass is estimated as $m_{v} \lesssim 1 \mathrm{eV}$ the quantity in the right-hand side of this inequality is close to the Plank energy.

In our calculations we used the approach based on the static value for the anomalous magnetic moment of neutrino. Unfortunately, the conditions of applicability of this approach at high energies are not known. However we believe that the results obtained in the present paper may provide some hints to possible future observation of the effects that are due to coherent neutrino interaction with a dense matter. The special feature of the results obtained in the present paper is that the pair production rate strongly depends on the helicity of the particles, and this leads to evident spatial asymmetries in the reaction products, which may have certain consequences observable in astrophysical and cosmological studies.

The author is grateful to A. V. Borisov, A. E. Shabad, and V. Ch. Zhukovsky for fruitful discussions.

This work was supported in part by the grant of President of Russian Federation for leading scientific schools (Grant SS - 5332.2006.2). 


\section{References}

[1] K. Fujikawa, R. Shrock, Phys. Rev. Lett. 45 (1980) 963.

[2] A. Lobanov, A. Studenikin, Phys. Lett. B 564 (2003) 27, hep-ph/0212393.

[3] A. Lobanov, A. Studenikin, Phys. Lett. B 601 (2004) 171, astro-ph/0408026

[4] V.A. Bordovitsyn, I.M. Ternov, V.G. Bagrov, Physics - Uspekhi 38 (1995) 1037, Uspekhi Fiz. Nauk 165 (1995) 1084.

[5] A. Studenikin, A. Ternov, Phys. Lett. B 608 (2005) 107, hep-ph/0410297, hep-ph/0412408.

[6] A.E. Lobanov, Dokl. Phys. 50 (2005) 286, Dokl. Akad. Nauk. Ser. Fiz. 402 (2005) 475, hep-ph/0411342.

[7] A.E. Lobanov, Phys. Lett. B 619 (2005) 136, hep-ph/0506007.

[8] A. Grigoriev, A. Studenikin, A. Ternov, Phys. Lett. B 622 (2005) 199, hep-ph/0502231, hep-ph/0507200.

[9] G.G. Raffelt, Stars as Laboratories for Fundamental Physics, Chicago, University of Chicago Press (1996).

[10] L. Wolfenstein, Phys. Rev. D 17 (1978) 2369.

[11] S.P. Mikheyev, A.Yu. Smirnov, Sov. J. Nucl. Phys. 42 (1985) 913, Yad. Fiz. 42 (1985) 1441.

[12] L.L. Foldy, Phys. Rev. 87 (1952) 688.

[13] P.B. Pal, T.N. Pham, Phys. Rev. D 40 (1989) 259.

[14] J.F. Nieves, Phys. Rev. D 40 (1989) 866.

[15] D. Nötzold, G. Raffelt, Nucl. Phys. B 307 (1988) 924.

[16] H. Bateman, A. Erdelyi, Higher transcendental functions, v. 3, New York-Toronto-London, McGraw-Hill Book Company, Inc. (1955).

[17] V.Ch. Zhukovsky, A.E. Lobanov, E.M. Murchikova, Phys. Rev. D 73 (2006) 065016, hep-ph/0510391.

[18] S. M. Carroll, G. B. Field, R. Jackiw, Phys. Rev. D 41 (1990) 1231. 
[19] D. Colladay, V. A. Kostelecký, Phys. Rev. D 55 (1997) 6760, hep-ph/9703464

[20] D. Colladay, V. A. Kostelecký, Phys. Rev. D 58 (1998) 11602, hep-ph/9809521.

[21] S. Coleman, S. L. Glashow, Phys. Rev. D 59 (1999) 116008, hep-ph/9812418. 